\title{
A comparison of mono-seasonal and multi- seasonal Landsat images for vegetation cover classification in the Mediterranean region: a case study in Latakia, Syria
}

\author{
Vasiliy Malinnikov ${ }^{1}$, and Assem Khatib $^{1,2^{*}}$ \\ ${ }^{1}$ Moscow State University of Geodesy and Cartography, Moscow 105064, Russia \\ ${ }^{2}$ Tishreen University, Lattakia, Syria
}

\begin{abstract}
Providing constantly updated information on vegetation serves as a basis for studies of natural resources and ecological issues. This paper discusses the question related to an appropriate season(s) for classification vegetation cover in the Mediterranean region and detecting its changes using Landsat imagery. Autumn, spring, and multi-seasonal satellite images, captured in 2017, were used to classify vegetation cover in a part of the Lattakia province, Syria. The satellite images were classified using the random forest algorithm, and high spatial resolution satellite images Google Earth Pro were used as reference data. The results indicate better effectiveness of the autumn images over spring ones for vegetation cover classification with $73.6 \%$ and $62.4 \%$ overall accuracy, respectively. In addition, a comparison of autumn and multi-seasonal Landsat images indicates no significant statistical difference in the accuracy of vegetation cover classification at the significance level of 0.05 , which illustrates the effectiveness of using autumn images to classify the vegetation cover of the Mediterranean region. Furthermore, the obtained results show the necessity of using additional features as the spectral channels may not be sufficient for mapping vegetation cover in the Mediterranean region with high accuracy.
\end{abstract}

\section{Introduction}

The vegetation cover of the Earth, being a source of valuable biological resources, simultaneously performs several functions as a regulator of fundamental processes of energy and substance exchange on the planet, plays an important ecological and sociocultural role for humanity [1]. The Mediterranean is one of the vital economic regions for agricultural and export of fruits purposes. The Mediterranean forests also play a valuable role as reservoirs of carbon and a source of biodiversity protection. The modern technical capabilities of Earth remote sensing systems from space make it possible to observe

\footnotetext{
* Corresponding author: syriaheart@live.com
} 
vegetation cover at global, regional, and local coverage depending on the different characteristics of these systems [2].

Landsat images are one of the most widely used data for mapping vegetation cover and detecting its changes for the following reasons: 1) High quality of geometric and radiometric corrections of Landsat data, which allows their use without the need for initial processing procedures. 2) It is possible to acquire free and accessible data of Landsat-5,7,8, homogeneous in terms of geometrical and radiometric characteristics, from 1984 to the present. 3) Planning the launch of Landsat-9 in 2021 with similar characteristics to Landsat8 ensures the further use of Landsat data [3].

Multi-season Landsat images are widely used in scientific research to increase the accuracy of vegetation cover mapping. However, the main problem with this approach lies in the difficulty of obtaining homogeneous multi-season images in different years, which leads to restrictions in the use of multi-season images to detect changes in vegetation cover.

When planning to map vegetation cover and detect its changes, the following critical questions arise 1) which seasons are appropriate to classify vegetation cover; 2) does the use of multi-season images increase the accuracy of vegetation cover classification compared to mono-seasonal images. Finding answers to these questions is the main objective of the research.

\section{Materials and Methods}

\subsection{Study Area}

The study area is part of the Lattakia province (Fig 1), located in the western region of Syria on the Mediterranean Sea coast. The study area occupies 80596 hectares. The climate of the study area is the Mediterranean, which is characterized by hot and dry in summers, wet and cool in winters [4]. The number of non-cloudy days is more frequent in autumn and spring compared to winter and summer. That is why autumn and spring images are more desirable for mapping and detecting changes in vegetation cover. Forests, citrus, and olive orchards constitute the main categories of vegetation cover in the Mediterranean region.

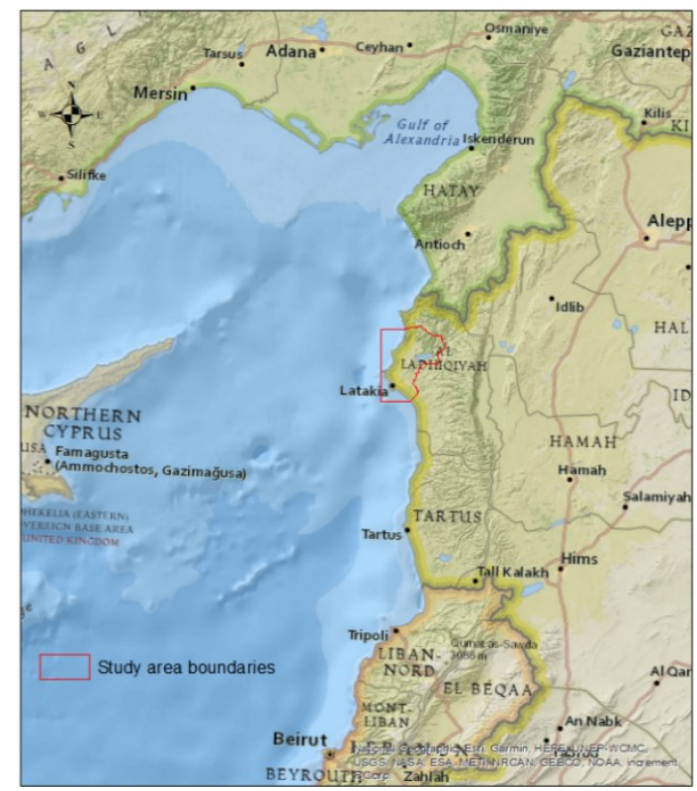


Fig 1. Location of the study area on the National Geographic World Map.

\subsection{Data}

In this study, Landsat-8 images of the study area, captured on the dates of March 25, 2017 (spring image) and October 19, 2017 (autumn image), were obtained from Earth Explorer (https://earthexplorer.usgs.gov/) with standard processing (Level-2). The six commonly used bands, that are blue (B: $\lambda=0.452-0.512 \mu \mathrm{m})$, green $(\mathrm{G}: \lambda=0.533-0.590 \mu \mathrm{m})$, red $(\mathrm{R}$ : $\lambda=0.636-0.673 \mu \mathrm{m})$, near-infrared (NIR: $\lambda=0.851-0.879 \mu \mathrm{m})$, and short wave infrared $\left(\mathrm{SWIR}_{1}: \lambda=1.566-1.651 \mu \mathrm{m} ; \mathrm{SWIR}_{2}: \lambda=2.107-2.294 \mu \mathrm{m}\right)$ bands with a spatial resolution of $30 \mathrm{~m}$ were used for vegetation cover classification. Examination of the intersection points of the streets on the spring and autumn images showed high accuracy in the geometric correction with an error of less than half a pixel, which indicates that there is no need for additional geometric corrections. Satellite images of Google Earth Pro with detailed spatial resolution, acquired in 2017-2018, were used as reference data.

\subsection{Vegetation cover classification using mono-seasonal and multi-seasonal Landsat images}

On the Landsat satellite data, six general categories of land cover were distinguished, including citrus orchards, olive orchards, forests, other types of vegetation (herbaceous and shrubby vegetation, winter crops), impervious surfaces (settlements, sand, exposed rocks), and water. A training sample, balanced for all categories (table 1), was formed based on the visual interpretation of Google Earth Pro satellite images. The training sample was distributed to represent the variation within each class and throughout the image.

Table 1. Distribution of the training sample for each category.

\begin{tabular}{|c|c|c|}
\hline Category & Designation & The number of training pixels \\
\hline Citrus orchards & C & 2211 \\
\hline Olive orchards & O & 2253 \\
\hline Forests & F & 2126 \\
\hline Other vegetation & Oth & 2302 \\
\hline Impervious surfaces & I & 2243 \\
\hline Water & W & 2314 \\
\hline
\end{tabular}

Autumn, spring, and multi-season (autumn and spring) images were independently classified using the same training sample. A random forest (RF) algorithm was applied to classify vegetation cover. RF is an ensemble classifier that uses a set of Decision Trees (DTs) classifiers to assign a final class for each pixel by majority voting of all the trees [5]. The following parameters were adopted for the random forest algorithm: the number of trees is 100; the number of variables in each tree is 3 for classifying the spring and autumn images, and 4 for the multi-season images.

A simple random sample with 670 referenced points, not located on the water category to avoid bias in the classification accuracy, was formed to assess and compare the classification of the vegetation cover based on mono-seasonal and multi-seasonal satellite images. Confusion matrix and statistics of accuracy (overall accuracy; user's accuracy; producer's accuracy) were calculated for each implemented classification according to the following equations [6]: 


$$
\begin{gathered}
O=\sum_{i=1}^{r} \frac{x_{i i}}{N} \\
U=\sum_{i=1}^{r} \frac{x_{i i}}{x_{i+}} \\
P=\sum_{i=1}^{r} \frac{x_{i i}}{x_{+i}}
\end{gathered}
$$

Where: $O$ - Overall accuracy; $U$ - User's accuracy; $P$ - Producer's accuracy; $N$ - sample size; $r$ - number of categories; $\mathrm{x}_{\mathrm{ii}}$-the number of reference points in row $i$ and column $i$ in the error matrix; $x_{i+}$ and $x_{+i}-$ The sum of reference points in row $i$ and column $i$, respectively.

The statistical test McNema was applied to assess the statistical significance of differences in the accuracy of vegetation cover classification in investigated cases at the significance level of 0.05 based upon the standardized normal distribution according to the formula [7]:

$$
z=\frac{f_{12}-f_{21}}{\sqrt{f_{12}+f_{21}}}
$$

Where: $f_{12}$ is the number of reference points, correctly classified using the first set of data and incorrectly classified using the second set; $\mathrm{f}_{21}$ is the number of control points, misclassified using the first set of data and correctly classified using the second.

\section{Results and Discussion}

The results of the vegetation cover classification using spring, autumn, and multi-seasonal Landsat images are presented in Fig 2.
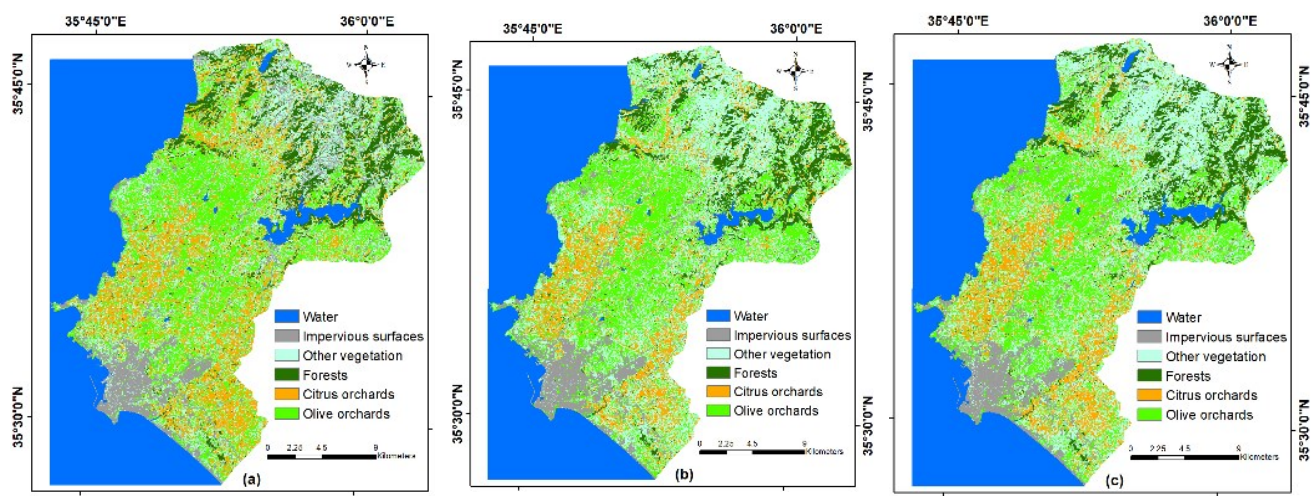

Fig 2. Vegetation cover classification using (a) spring, (b) autumn, and (c) multi-seasonal Landsat images. 
Tables 2-4 provide error matrices and accuracy statistics of vegetation cover classification using spring, autumn, and multi-seasonal satellite images.

Table 2. Error matrix of classification using spring satellite images.

\begin{tabular}{cccccccc}
\hline Classified & \multicolumn{7}{c}{ Reference Data } \\
\cline { 2 - 7 } Data & $O$ & $C$ & $F$ & Oth & $I$ & Total & $U \%$ \\
$O$ & 120 & 9 & 1 & 54 & 3 & 187 & 64.2 \\
$C$ & 12 & 98 & 11 & 45 & 1 & 167 & $\mathbf{5 8 . 7}$ \\
$F$ & & 8 & 84 & 10 & & 102 & 82.4 \\
Oth & 51 & 15 & 21 & 107 & 10 & 204 & 52.5 \\
$I$ & 6 & & & 32 & 75 & 113 & 66.4 \\
$W$ & & & & 1 & 2 & 3 & \\
Total & 189 & 130 & 117 & 249 & 91 & 776 & \\
$P \%$ & $\mathbf{6 3 . 5}$ & 75.4 & $\mathbf{7 1 . 8}$ & $\mathbf{4 3 . 0}$ & 82.4 & & $O=62.4 \%$ \\
\hline
\end{tabular}

Table 3. Error matrix classification using autumn satellite images.

\begin{tabular}{cccccccc}
\hline Classified & \multicolumn{7}{c}{ Reference Data } \\
\cline { 2 - 7 } Data & $O$ & $C$ & $F$ & Oth & $I$ & Total & $U \%$ \\
$O$ & 137 & 5 & 3 & 53 & 4 & 202 & $\mathbf{6 7 . 8}$ \\
$C$ & 3 & 99 & 4 & 20 & & 126 & 78.6 \\
$F$ & & 7 & 99 & 6 & & 112 & 88.4 \\
Oth & 45 & 19 & 10 & 154 & 5 & 233 & 66.1 \\
$I$ & 4 & & & 16 & 82 & 102 & 80.4 \\
$W$ & & & 1 & & & 1 & \\
Total & 189 & 130 & 117 & 249 & 91 & 776 & \\
$P \%$ & 72.5 & $\mathbf{7 6 . 2}$ & $\mathbf{8 4 . 6}$ & $\mathbf{6 1 . 8}$ & 90.1 & & $O=73.6 \%$ \\
\hline
\end{tabular}

Table 4. Error matrix of classification using multi-seasonal satellite images.

\begin{tabular}{cccccccc}
\hline Classified & \multicolumn{7}{c}{ Reference Data } \\
\cline { 2 - 7 } Data & $O$ & $C$ & $F$ & Oth & $I$ & Total & $U \%$ \\
$O$ & 135 & 5 & 3 & 46 & 4 & 193 & $\mathbf{6 9 . 9}$ \\
$C$ & 5 & 107 & 2 & 18 & & 132 & $\mathbf{8 1 . 1}$ \\
$F$ & & 3 & 100 & 5 & & 108 & 92.6 \\
Oth & 44 & 14 & 12 & 168 & 2 & 240 & 70.0 \\
$I$ & 5 & 1 & & 11 & 83 & 100 & 83.0 \\
$W$ & & & & 1 & 2 & 3 & - \\
Total & 189 & 130 & 117 & 249 & 91 & 776 & \\
$P \%$ & 71.4 & 82.3 & $\mathbf{8 5 . 5}$ & $\mathbf{6 7 . 5}$ & 91.2 & & $O=76.4 \%$ \\
\hline
\end{tabular}


The best results to classify the category (forests) were obtained using multi-seasonal satellite images with a minimum accuracy (minimum between user's and producer's accuracy) of $85.5 \%$, followed by autumn images $-84.6 \%$, and then spring ones $-71.8 \%$. These results indicate a significant improvement of $12.8 \%$ in the accuracy of the category (forests) using autumn images compared to spring images. Results also show an improvement in the accuracy of $0.9 \%$ using multi-seasonal images compared to autumn images.

The best results to classify the category (citrus orchards) were obtained using multiseasonal satellite images with a minimum accuracy of $81.1 \%$, followed by autumn images $76.2 \%$, and then spring ones $-58.7 \%$. These results indicate a significant improvement of $17.5 \%$ in the accuracy of the category (citrus orchards) using autumn images compared to spring images. Results also show an improvement in the accuracy of $4.9 \%$ using multiseasonal images compared to autumn images.

The best results to classify the category (olive orchards) were obtained using multiseasonal satellite images with a minimum accuracy of $69.9 \%$, followed by autumn images $67.8 \%$, and then spring ones $-63.5 \%$. These results show an improvement of $4.3 \%$ in the accuracy of the category (olive orchards) using autumn images compared to spring images. Results also show an improvement in the accuracy of $2.1 \%$ using multi-seasonal images compared to autumn images.

The best results to classify the category (other vegetation) were obtained using multiseasonal satellite images with a minimum accuracy of $67.5 \%$, followed by autumn images $61.8 \%$, and then spring ones $-43.5 \%$. These results indicate a significant improvement of $18.3 \%$ in the accuracy of the category (other vegetation) using autumn images compared to spring images. Results also show an improvement in the accuracy of $5.7 \%$ using multiseasonal images compared to autumn images.

The overall accuracy of vegetation cover classification is $73.6 \%$ when using autumn satellite images, while it is $62.4 \%$ when using spring ones. These results indicate better effectiveness of the autumn images over spring ones for vegetation cover classification. Furthermore, the calculated value of $|z|=5.36$ is higher than $|z|=1.95$ - the tabulated value. These results indicate a significant statistical difference in the accuracy of using autumn and spring satellite images for vegetation cover classification at the significance level of 0.05 . The lower classification accuracy using spring images is explained by the matrix of errors that there is significant confusion between the category (forest) and the category (other vegetation) on the one hand, and the categories (olive and citrus orchards) and the category (other vegetation) on the other. The growth of grass and herbs and the cultivation of winter crops in orchards in the spring are possible causes of vegetation cover classification errors to increase using spring images.

The best results to classify the vegetation cover in the study area were obtained using multi-seasonal satellite images with an overall accuracy of $76.4 \%$, followed by autumn images $-73.6 \%$, and then spring ones $-62.4 \%$. These results indicate better effectiveness of the multi-seasonal satellite images over spring ones. There is also a slight increase in accuracy of the multi-seasonal satellite images over autumn ones for vegetation cover classification. In the latter case, the calculated value of $|z|=1.91$, less than 1.95 . These results indicate no significant statistical difference in the accuracy of using autumn and multi-seasonal satellite images for vegetation cover classification at the significance level of 0.05 . 
Our finding that multi-seasonal Landsat images give greater accuracy in the classification of vegetation cover than mono-seasonal images is in agreement with the results of the studies conducted in different regions of the world [8-10]. In works [11-13] was investigated the selection of the appropriate algorithm and features for the classification of land cover, including the vegetation in the Mediterranean region, using Landsat images. The findings of these studies indicate the effectiveness of the random forest algorithm for land cover classification in the Mediterranean region using satellite images, so in this study, this algorithm was used.

This study, as in work [14], was investigated the selection of the appropriate season/seasons for the classification of vegetation cover in the Mediterranean region. The study [14] showed that the best minimum accuracy to classify the categories (cereal, grassland, and shrub) was obtained using multi-seasonal satellite images, followed by autumn images, and then spring ones. That study also showed that the best minimum accuracy to classify the category (wood) was obtained using autumn satellite images, followed by multi-seasonal images, and then spring ones. In our study, classes (cereal, grassland, and shrub) were grouped into one category (other vegetation) due to the small field area for these classes in the study area, which made it difficult to distinguish them within independent categories. In addition, the category (wood) was divided into three categories (forests, citrus orchards, and olive orchards). The accuracy of using multi-season images to classify these categories is slightly higher than the autumn images, but it is much higher than the spring images. This comparison indicates a significant effect of the studied landscape on selecting the appropriate season/seasons when using Landsat images for vegetation cover classification.

\section{Conclusion}

The main objective of this paper is to compare the results of vegetation cover classification in the Mediterranean region using mono-seasonal and multi-seasonal Landsat images. A random forest algorithm was applied to classify vegetation cover using Landsat images. The overall accuracy of vegetation cover classification was $73.6 \%$ using autumn images, $62.4 \%$ using spring images, and $76.4 \%$ using mono-seasonal images. The statistical test McNema was applied, and the value of $|z|$ was calculated. Results indicate a significant statistical difference in the accuracy of using autumn and spring satellite images for vegetation cover classification at the significance level of 0.05 . Additionally, the results indicate no significant statistical difference in the accuracy of using autumn and multiseasonal satellite images for vegetation cover classification.

According to the analysis of the results, it can also, concluded that using spectral bands of mono-seasonal Landsat images may not be sufficient to obtain a high accuracy of vegetation cover classification with more than $80 \%$ in complex Mediterranean landscapes. Research recommendations can include using autumn Landsat images to classify the vegetation cover of the Mediterranean region and using additional features such as topographic and texture features to increase the reliability of classification.

\section{References}

1. S. A. Bartalev, V. A. Egorov, V. O. Zharko, E. A. Loupian, D. E. Plotnikov, S. A. Khvostikov, Sovr. Probl. DZZ Kosm 12(5), 203-221 (2015) 
2. S. A. Bartalev, V. A. Egorov, V. O. Zharko, E. A. Loupian, D. E. Plotnikov, S. A. Khvostikov, N. V. Shabanov, Land cover mapping over Russia using Earth observation data (IKI, Moscow, 2016)

3. M. Wulder, et al, Remote Sens. Environ, 225, 141 (2019)

4. A. Khatib, V.A. Malinnikov, Sovr. Probl. DZZ Kosm, 18(4), 115-127 (2021)

5. M. Belgiu, L. Dragut, ISPRS J. Photogramm. Remote Sens, 114, 26 (2016)

6. R. Congalton, K. Green, Assessing the Accuracy of Remotely Sensed Data: Principles and Practices (CRC Press, Florida, 2009)

7. G. Foody, PHOTOGRAMM ENG REM S, 70, 630 (2004)

8. D. E. Plotnikov, P. A. Kolbudaev, S. A. Bartalev, E. A. Loupian, Sovr. Probl. DZZ Kosm 15(2), 112-127 (2018)

9. E.A. Stytsenko, Sovr. Probl. DZZ Kosm 14(5), 172-183 (2017)

10. V. Rodriguez-Galiano, M. Chica-Rivas, Int. J. Digit. Earth 7, 5 (2012)

11. A. Khatib, V.A. Malinnikov, Sovr. Probl. DZZ Kosm 18(2), 51-63 (2021)

12. V. Rodriguez-Galiano., B. Ghimire., J. Rogan, M. Chica-Olmo, J. Rigol-Sanchez, SPRS Ann. Photogramm. Remote Sens, 67, 93-104 (2012)

13. D. Gounaridis, A. Apostolou, S. Koukoulas, J. Maps, 12(5), 1055-1062 (2016)

14. C. Senf, P. Leitao, D. Pflugmacher, S. Linden, P. Hostert, Remote Sens. Environ, 156, 527-536 (2014) 